\title{
Silent Industrial Leap for Relatively Unknown Optical Elements
}

\section{Divliansky IB*}

Department of Photo-Induced Processing, College of Optics and Photonics - CREOL, University of Central Florida, Orlando, FL, USA

In the past decade, one can say almost covertly, a specific type of optical elements has found their places in broad area of laser systems, devices and optical systems as a whole. These elements are called volume Bragg gratings or VBGs for short. They slowly claimed their ground and now are manufactured in thousands and incorporated from simple laser diode modules to complex femtosecond systems. The mergers of laser companies and in general the globalization of the lasers and optics markets in the past decade helped a lot for this silent, but also big leap in the number of VBGs used in commercial as well as research applications.

The simplest volume Bragg grating is a system of planar layers with modified refractive index. Depending on the diffraction angle and the orientation of the grating in the plate, one can distinguish several types of Bragg gratings (Figure 1). A grating is called a transmitting Bragg grating if the diffracted beam crosses the back surface, reflecting - if the diffracted beam crosses the front surface, and prismatic - if the diffracted beam crosses one of the side surfaces.

These are the most basic types of VBGs but regardless they are widely used as wavelength selective mirrors or angular filters. For example, reflective VBGs (RBGs) are used for narrowing the spectrum of laser diode pump sources. When directly used, pump diodes have emission linewidth of several or more nanometers which even though is much better than flash lamp pumping it is still an inefficient way of exciting a laser medium. In the recent years, many companies manufacturing diode laser for pumping, started to lock the diode sources in an external resonator scheme using narrow line VBGs bringing the pump spectrum to tens of picometers or below. Given the number of diode pumped laser systems that are sold every year across the world, one can easily see the enormous number of VBGs employed only for this application. Reflecting VBGs are also used, very frequently, as standard dichroic or filtering element in research laser system. The fact that reflective volume gratings can be made to have very narrow spectral widths makes them also ideal as notch filters. The state of the art Bragg notch filters can reflect bandwidth of only $5 \mathrm{~cm}-1$ while transmitting the rest of the spectrum. These VBGs are ideal as filters for Raman spectroscopy and have been widely implemented in Raman instruments.

For any application, it is quite important to have the VBG recorded in medium that has very good optical properties. Under very good is understood material with high optical uniformity (minimal refractive index fluctuations), low absorption and low scattering. There are many VBG host materials available but the one that stands out and is the most widely used for recording VBGs that are used in the industry and most research labs is called photo-thermo refractive glass. Its development in the past few years allowed the recording of high quality
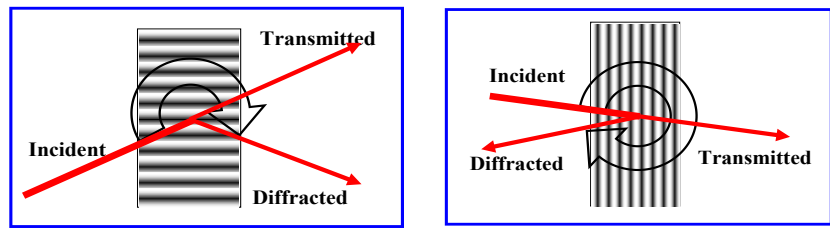

Figure 1: Transmitting (left) and reflective (right) volume Bragg gratings. The VBG occupies the whole volume of the medium. simple VBGs as well as the fabrication of previously technologically challenging complex VBGs. Prime example of such complex gratings are the longitudinally chirped ones which most of the time are just call "Chirped VBGs or CVBGs". Such gratings have period that change along the beam propagation direction which corresponds to varying of the Bragg wavelength along the length of the VBG. If one can imagine a broad-spectrum laser pulse entering such VBG he will see that different spectral parts of the pulse will be reflected after propagating different lengths of the grating (Figure 2). As a result, the reflected pulse will be stretched in time because its spectral parts were reflected after propagating different paths in the glass.

Obviously, these kinds of volume gratings are perfect for stretching and if used in reverse, for compressing ultrashort pulses. They have key benefits that make them superior to surface gratings when performing the same task. Surface gratings are easily damaged or contaminated, and cannot sustain high power levels in most cases. On the other hand, to achieve enough stretching using CVBG, a volume grating with a length on order of $15 \mathrm{~mm}$ or more is required. This is where the quality of the glass used to record the gratings into plays enormous role. If the glass has slightly varying refractive index, the stretching of the ultrashort pulse will not be uniform due to the non-uniformity of the Bragg grating. Upon amplification and recompression, the pulse will not be recompressed back to its initial parameters. This is especially detrimental in commercial femtosecond systems, given the fact that some of which are used as medical devices for cataract eye surgeries.

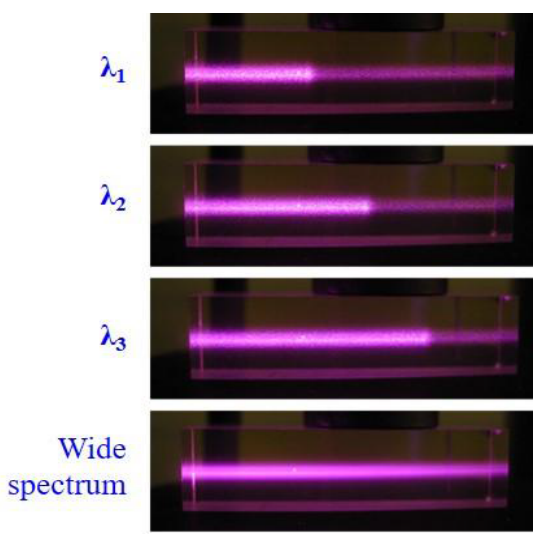

Figure 2: Demonstration of the propagation length of different wavelengths when reflected by a chirped VBG.

*Corresponding author: Divliansky IB, Department of Photo-Induced Processing College of Optics and Photonics - CREOL, University of Central Florida, Orlando, FL, USA, Tel: 110-407-823-6827; E-mail: ibd1@creol.ucf.edu

Received August 25, 2017; Accepted August 29, 2017; Published September 25, 2017

Citation: Divliansky IB (2017) Silent Industrial Leap for Relatively Unknown Optical Elements. J Laser Opt Photonics 4: e113. doi: 10.4172/2469-410X.1000e113

Copyright: (c) 2017 Divliansky IB. This is an open-access article distributed under the terms of the Creative Commons Attribution License, which permits unrestricted use, distribution, and reproduction in any medium, provided the original author and source are credited. 
Citation: Divliansky IB (2017) Silent Industrial Leap for Relatively Unknown Optical Elements. J Laser Opt Photonics 4: e113. doi: 10.4172/2469-410X.1000e113

Page 2 of 2

This currently is one of the largest markets for chirped volume Bragg gratings - their use as stretcher/compressor in medical ultrashort pulse systems.

An example and proof of how important VBGs have become in the last ten years is the recent purchase of the biggest manufacturer of VBGs (OptiGrate Corp.) by one of the largest laser companies in the world - IPG Photonics. IPG Photonics is world leader in high-power fiber lasers and amplifiers and the acquisition of OptiGrate will greatly benefit their new line of ultrafast-pulsed lasers.

Other types of VBGs such as transmitting multiplexed, Moiré, focusing/defocusing, and others, have their own unique applications where they are the preferred specialty optics. As in the cases of selective mirrors and chirped VBGs, they steadily prove their superiority to their standard optical alternatives in performance, damage tolerances and handling. I expect that their market share will steadily grow in the near future and that they will be used in even more devices and research areas.

As final remark I can say, that VBGs surprisingly without any noise overtook the positions of certain traditional optical elements that have been in use for decades. That trend for sure will continue with the further development of certain photosensitive glasses that will open new venues for complex VBGs in the visible and the near-IR. 